\title{
Wofür wir uns schämen
}

\section{Thesen zu den Funktionen des Privaten in den Boulevardmedien. Von Friederike Herrmann}

Prof. Dr. Friederike Herrmann ist Professorin für Journalistik und Kommunikationswissenschaft an der $\mathrm{Ka}$ tholischen Universität Eichstätt-Ingolstadt.
Abstract Wenn pauschal vom Privaten in den Medien gesprochen wird, geht leicht verloren, wie komplex der Begriff Privatheit eigentlich ist. Der Beitrag unterscheidet drei Kategorien des Privaten in den Boulevardmedien am Beispiel von „Bild“: Erstens die voyeuristische Berichterstattung, die unzulässig in die Privatsphäre der Akteure eindringt. Die Berichte der zweiten Kategorie stellen gesellschaftspolitisch relevante Themen dar. Sie erfüllen klassische journalistische Funktionen, thematisieren häufig aber auch persönliche Erfahrungen und Gefühle der jeweiligen Akteure. Damit stehen sie im Widerspruch zu einem Gesellschaftsmodell, das Öffentlichkeit und Privatheit dichotomisch versteht. Die dritte Kategorie der Beiträge ist noch kaum untersucht. Sie schildern alltägliche Begebenheiten und skandalisieren dabei kaum. Analysen zeigen, dass in diesen Beiträgen sehr häufig Gefühle der Scham verhandelt werden. Viele der Beiträge stellen die Akteure in beschämenden Situationen dar, ohne ein Identifikationsangebot zu machen. Möglicherweise dient das der Delegation von Schamgefühlen der Rezipienten. Aus medienethischer Perspektive ist eine solche öffentliche Beschämung abzulehnen.

W er die Seiten der „Bild“-Zeitung nach privaten Themen durchforstet, wird feststellen, wie unglaublich banal viele dieser Berichte sind. Da erzählt Schauspieler Bryan Cranston, dass er leidenschaftlich gerne die Spülmaschine einräumt. Irgendwo in Berlin wurde das Hündchen einer Frau überfahren, die nun untröstlich ist. Eine 36-köpfige Hochzeitsgesellschaft feiert sparsam für 7,90 Euro pro Person beim Chinesen. In Berlins „Viertel der Verlierer“, dem Maulbergallee-Kiez, wird schon um 12 Uhr mittags Bier getrunken. Und Töchter finden ihre Mütter peinlich gekleidet, weshalb der Modedesigner Guido Maria Kretschmer den Müttern ein neues Outfit verpasst. ${ }^{1}$

1 Quelle dieser Berichte war jeweils die „Bild“-Zeitung. In der Reihenfolge der Aufzählung: Bundesausgabe 17.5.2014; Regionalausgabe Berlin 16.5.2014; ebd. 17.5.2014; ebd.; Bundesausgabe 14.5.2014. 
Diese Geschichten spotten jeder Nachrichtenwerttheorie (vgl. z. B. Meier 2013, 198ff). Die Akteure sind nur selten Prominente, es wird kaum emotionalisiert oder skandalisiert, weder Sex noch Crime spielen eine wesentliche Rolle. Auch die gängigen Erklärungen für private Themen in den Medien greifen in diesen Fällen nicht: Weder soziale Orientierung noch Rat und Hilfe bieten diese Berichte wirklich an, und der Unterhaltungswert scheint eher gering. Warum also erreichen diese Themen ein Millionenpublikum? Welchen Nutzen, welchen Gewinn ziehen die Leserinnen und Leser daraus? Die „Bild“-Zeitung orientiert sich radikal am Publikumsgeschmack, geht also davon aus, dass diese Texte gerne gelesen werden.

Vom Privaten in den Medien wird häufig recht pauschal gesprochen. Dabei gerät leicht aus dem Blick, welch komplexer Begriff Privatheit ist und dass unter dieses Schlagwort unterschiedliche Dimensionen der Berichterstattung fallen. Ich will im Folgenden drei verschiedene Kategorien der Thematisierung des Privaten in den Medien nach ihrer Funktion unterscheiden ${ }^{2}$ : Erstens die Thematisierung des Privaten aus voyeuristischem Interesse, zweitens Berichte über Privates, die klassische journalistische Aufgaben erfüllen, indem sie Orientierung und Rat bieten oder zur Selbstverständigung und Selbstbeobachtung der Gesellschaft beitragen, und drittens die Wiedergabe scheinbar banaler Alltagserzählungen, wie in den oben zitierten Geschichten, deren Funktion noch weiterer Untersuchungen bedarf. Möglicherweise dienen sie der Delegation von Schamgefühlen, wie ich erläutern werde. Nicht immer sind journalistische Beiträge nur einer dieser Kategorien zuzuordnen, sie können auch mehrere Funktionen gleichzeitig erfüllen.

\section{Voyeurismus und politisch Relevantes}

Wenn die Berichterstattung in den Boulevardmedien kritisiert wird, steht meist der voyeuristische Blick auf das Private im Vordergrund. Die Opfer dieser Übergriffe sind häufig Prominente. So gaben beispielsweise Fotos der britischen Herzogin Kate

2 Ausgespart bleiben Themenbereiche, die nach allgemeiner Überzeugung unbestritten ihren Platz in der medialen Berichterstattung haben sollten und nur mehr oder weniger stark nach privatwirtschaftlichen Gesichtspunkten organisiert sind, wie private Medien, private Bildungsanstalten usw. Der Verweis zeigt aber, wie schillernd der Begriff des Privaten genutzt wird und wie wenig er pauschal als Maßstab für die Berichterstattung der Medien taugt. 
mit allzu hoch gewehtem Rock der „Bild am Sonntag“ reichlich Anlass für zotige Sprachspiele (Ausgabe vom 25.5.2014). Aber die Schelte der Boulevardmedien ist billig: Auch seriösere Zeitungen übernehmen solche Meldungen gerne für ihre bunte Seite. Medienethisch brisanter sind jedoch die Fälle, in denen beispielsweise ohnehin schon unter Schock stehende Angehörige von Unglücksopfern bis in ihre Wohnungen von Medien verfolgt werden und ihren Privatraum nicht mehr schützen können (vgl. Herrmann 2003). Der Pres-

Bürgerinnen und Bürger sollen selbst entscheiden dürfen, was sie von sich preisgeben, sofern kein berechtigtes öffentliches Interesse besteht. sekodex des Presserates formuliert zu diesen Fällen in Ziffer 8 eindeutig: „Die Presse achtet das Privatleben des Menschen und seine informationelle Selbstbestimmung." Hier geht es um den Schutz der Persönlichkeit, Bürgerinnen und Bürger sollen selbst entscheiden dürfen, was sie von sich preisgeben, sofern kein berechtigtes öffentliches Interesse besteht. Medienethisch ist dieser Schutz der Privatsphäre unstrittig.

Über Themen, die dem häuslichen und privaten Bereich zugerechnet werden, haben die Medien jedoch schon immer berichtet, ohne dass sie dabei die Privatsphäre unerlaubt verletzten. Diese Beiträge können durchaus zentrale journalistische Funktionen erfüllen. In diese Kategorie fällt beispielsweise eine Serie der „Bild“-Zeitung über Demenz, die viele nützliche Tipps enthält und durchaus auch einfühlsam von den vielfältigen Ausprägungen und den Erfahrungen der Betroffenen und ihrer Angehörigen mit dieser Krankheit berichtet (mehrere Ausgaben im Mai 2014). Solche Beiträge sind nicht nur praktische Lebenshilfe und Ratgeber - sie sind auch die Grundlage einer öffentlichen Meinungsbildung zum Thema, die Voraussetzung politischer Entscheidungen ist: Welche Einrichtungen und Forschungsfelder werden mit Steuergeldern gefördert, wie werden die Stufen der Pflegeversicherung bestimmt, welche Hilfen sollte die Gesellschaft anbieten? In solchen Artikeln muss auch ganz subjektiv aus dem Privaten berichtet werden: Ohne die Erfahrungen und Gefühle der Betroffenen sind die Dimensionen dieser Krankheit nicht vermittelbar. Doch an der Frage, ob Gefühle und Erfahrungen überhaupt an die Öffentlichkeit gehören, scheiden sich die Geister.

Es wäre allerdings ein sehr enger Politikbegriff, wollte man ein Parteiengezänk über Steuern für politischer halten als solche Auseinandersetzungen mit den Bereichen familiärer Pflege, Kindererziehung, Hausarbeit, Familienformen, Krankheit usw. 
Tatsächlich aber werden diese Themen im Politikressort selten aus der Perspektive des Alltags der Bürgerinnen und Bürger betrachtet, sondern weit häufiger aus der Perspektive der Institutionen von Politik und Gesellschaft. Dies hat auch mit den Begriffen des Privaten und Öffentlichen in unserer Gesellschaft zu tun.

\section{Die Debatte um Privatheit und Öffentlichkeit}

Wissenschaftlerinnen und Feministinnen setzen sich seit Jahrzehnten kritisch mit der Idee auseinander, dass Öffentlichkeit und Privatheit in unserer Gesellschaft dichotomisch zu verstehen seien. ${ }^{3}$ Die Frauenbewegung der 1970er Jahre hat dies mit dem griffigen Slogan „Das Private ist politisch“ zur Diskussion gestellt. Der Dualismus des Privaten und Öffentlichen, so die Kritik, habe historisch zur Ausgrenzung der Frauen und ihrer Interessen aus Politik und Öffentlichkeit geführt. Er verschleiere zudem, wie stark politische Entscheidungen von privaten Erfahrungen beeinflusst seien. Und er habe Täter im privaten Bereich weitgehend der öffentlichen Kontrolle entzogen. Erst in der Folge dieser Debatten wurde beispielsweise Vergewaltigung in der Ehe unter Strafe gestellt und Missbrauch in Familien zu einem öffentlich diskutierten Thema.

Eine solche öffentliche Relevanz ist für die dritte Kategorie privater Themen in den Boulevardmedien nicht erkennbar. $\mathrm{Ob}$ ein Schauspieler die Spülmaschine einräumen mag, Töchter ihre Mütter schlecht gekleidet finden oder in Berlin ein Hund überfahren wird, muss die Gesellschaft nicht interessieren. Hinzu kommt, dass die Erzählweise dieser Beispiele ausgesprochen unaufgeregt ist

Der Boulevardjournalismus verhandelt - ähnlich wie die Literatur-Gefühle, die das Publikum aus dem eigenen Alltag kennt. und nicht so sensationsheischend, wie man es von einer Boulevardzeitung vielleicht erwartet hätte. Warum also bringt die „Bild“-Zeitung in den meisten Ausgaben mehrere solcher harmlosen Geschichten, warum goutieren die Leser dies?

Eine Erklärung könnte sein, dass in diesen Texten, ähnlich wie in der Literatur, Gefühle verhandelt werden, die das Publikum aus dem eigenen Alltag kennt (vgl. Mellmann 2011). Analysiert man die Geschichten unter dieser Fragestellung, so lässt 
sich vor allem ein Gefühl herausarbeiten, das die Artikel bearbeiten: das Gefühl der Scham. ${ }^{4}$

Ein Beispiel: Auf den ersten Blick klingt der oben zitierte, groß aufgemachte Artikel über eine Frau, deren Hund überfahren wurde, nach einer etwas rührseligen Geschichte zum Mitweinen. Untersucht man die Szene, die der Text entwirft, jedoch genauer, ist eine geradezu gegenteilige Lesart plausibler: Da zeigt jemand ein beschämendes Verhalten. Die Frau wirft sich auf die Straße, ruft „Meine Frieda, meine Frieda“, gibt sich untröstlich und muss von Polizisten mühsam auf den Gehweg zurückgeholt werden. Die Leser erfahren, dass sie den Hund (Frieda) nicht angeleint hatte, er auf die Straße lief und eine Busfahrerin keine Chance mehr hatte zu bremsen. Nichts wird hingegen über die Beziehung der Frau zu ihrem Hund erzählt, was die Voraussetzung einer empathischen Identifikation mit der Frau und ihren Gefühlen wäre und ihr Weinen verständlicher machen könnte. Stattdessen erfahren wir, dass sie einen Fehler gemacht, versagt hat, indem sie das Tier nicht anleinte. In der von der „Bild“-Zeitung dargestellten Szene lässt sich die Reaktion der Hundebesitzerin als unangemessen und hysterisch interpretieren, zumal mit dem Hundenamen „Frieda“ an das Stereotyp von Frauen angeknüpft wird, die ihre Hunde wie Kinder behandeln. Studierende, denen ich den Text vorgelegt habe, reagierten mit Äußerungen wie „Hat die keine anderen Sorgen“ und „selber schuld, wenn sie den Hund nicht anleint". ${ }^{5}$ Die Situation kann also vom Betrachter eher als beschämend für die Frau gelesen werden.

Die Hundebesitzerin selbst verspürt anscheinend keine Schamgefühle. Hier erhält das hippe Wort vom Fremdschämen eine interessante Umkehrung: Gemeint wäre dann gerade nicht das empathische Mitleiden mit einem Menschen, der sich schämt, sondern das Vorführen einer Person, die anschei-

4 Ich habe die Texte nach einem Verfahren szenischen Verstehens analysiert, das ich an Konzepte Alfred Lorenzers (1973) und Erving Goffmans (1959/2011) angelehnt habe. Das Verfahren untersucht die Interaktionen zwischen Akteuren, impliziter Erzählinstanz und impliziten Rezipienten des Textes. Vorarbeiten zu dieser Methode habe ich an anderer Stelle dargestellt (Herrmann 2013), eine Veröffentlichung, die die Methode ausführlich beschreibt, ist in Planung.

5 Den Text habe ich einer Gruppe von elf Studierenden vorgelegt. Zehn von ihnen fanden die Reaktion der Frau unangemessen. Nur eine Studentin äußerte Verständnis. Sie ist selbst Hundebesitzerin und konnte sich deshalb vermutlich einfühlen, obwohl der Text dafür kein Angebot macht. 
nend nicht weiß, wofür sie sich zu schämen hat. Diese Beschämung des anderen könnte von den Leserinnen und Lesern zur eigenen Gefühlsregulation genutzt werden. Die in unserer Gesellschaft allgegenwärtige Angst, unversehens in beschämende Situationen zu geraten, wird in solchen Texten bearbeitet, indem die Leser sie auf die Akteure der Texte projizieren und so delegieren können. ${ }^{6}$

\section{Die Angst vor der Scham}

Scham ist ein Gefühl, das schwer zu ertragen ist. Das spiegelt sich in Redewendungen wieder, dass man vor Scham in den Boden versinken möchte. Lieber will man verschwinden als mit diesem Gefühl umgehen. Zwar bescheinigen manche Zeitdiagnostiker der Gegenwart einen Verlust an Schamgefühl, was stets als Kulturkritik gemeint ist. Vielleicht aber übersehen diese Kritiker nur, wie stark die Ursachen der Scham kultur- und zeitabhängig sind und wie rasch sie sich in unserer Gesellschaft wandeln. Psychologen weisen darauf hin, wie sehr heute bestimmte Dinge mit Scham besetzt sind: Versagen und Scheitern beispielsweise oder der nicht makellose, nicht durchtrainierte Körper (vgl. Herrmann 2003, S. 148f.). Gerade der rasche Wandel dessen, wofür man sich

Scham ist ein Gefühl, das schwer zu ertragen ist. Lieber will man in den Boden versinken und verschwinden als mit diesem Gefühl umgehen. zu schämen hat, schafft weitere Verunsicherung: Die Angst, die Regeln nicht zu kennen und unversehens in eine Situation zu geraten, die beschämend ist. Auch für das Nicht-Schämen kann man sich schämen (vgl. auch Greiner 2014).

Eine weitere Form, die Angst vor der Scham zu bearbeiten, ist in den vielen Texten enthalten, die beschreiben, wie normal erfolgreiche Menschen doch eigentlich sind. Zum Beispiel der Schauspieler Bryan Cranston, der sagt: „Mein Luxus ist es, nach Hause zu kommen und den Geschirrspüler einzuräumen." Am Ende des Interviews schaut Cranston auf den Ehering des Reporters und erklärt: „Wir Verheirateten müssen zusammenhalten.“ Wenn das so ist, dann müssen sich auch die Leserinnen und Leser nicht schämen, keine besonderen Wünsche und hehren Ziele zu haben, keine besonderen Partnerschaften zu leben, sondern nur spießig und normal zu sein.

6 Auf der Inhaltsebene habe ich nur untersucht, welches Angebot diese Texte an die Rezipientinnen und Rezipienten macht. Weitere Studien wären erforderlich um tatsächliche Lesarten herauszuarbeiten. 
Die privaten Geschichten in den Boulevardmedien lesen sich wie ein Kompendium dessen, wofür man sich in dieser Gesellschaft zu schämen hat: Es geht um Geiz und Gier, Lieblosigkeiten und Versäumnisse, Makel des äußeren Erscheinungsbildes, Versagen und Unfähigkeiten. ${ }^{7}$ Medienethisch bedenklich sind in diesem Zusammenhang insbesondere die Beiträge, die andere Menschen öffentlich beschämen. Viele Boulevardformate, gerade auch im Fernsehen, scheinen jedoch genau mit diesem Muster zu spielen. Sie entblößen Menschen in psychischer Hinsicht, was medienethisch sicher nicht weniger problematisch ist als ein nicht autorisiertes Nacktfoto.

\section{Literatur}

Goffmann, Erving (1959/2011): Wir alle spielen Theater. Die Selbstdarstellung im Alltag. München.

Greiner, Ulrich (2014): Schamverlust. Vom Wandel der Gefühlskultur. Reinbek. Herrmann, Friederike (2002): Privatheit, Medien und Geschlecht. Bisexualität in Daily Talks. Opladen.

Herrmann, Friederike (2003): Ein neuer Begriff des Privaten - Scham als medienethische Kategorie. In: Debatin, Bernhard/Funiok, Rüdiger (Hg.): Kommunikations- und Medienethik. Konstanz, S. 143-156.

Herrmann, Friederike (2013): Vom Oberlehrer zum Kumpel - Das Beziehungsgeflecht journalistischer Texte im Medienvergleich. In: Renner, Karl N./Hoff, Dagmar von/Krings, Matthias (Hg.): Medien. Erzählen. Gesellschaft. Transmediales Erzählen im Zeitalter der Medienkonvergenz. Berlin, S. 241-264.

Klaus, Elisabeth (2005): Kommunikationswissenschaftliche Geschlechterforschung. Zur Bedeutung der Frauen in den Massenmedien und im Journalismus. Aktualisierte und korrigierte Neuauflage. Wien.

Lorenzer, Alfred (1973): Sprachzerstörung und Rekonstruktion. Vorarbeiten zu einer Metatheorie der Psychoanalyse. Frankfurt am Main.

Meier, Klaus ( $\left.{ }^{2} 2013\right)$ : Journalistik. Konstanz.

Mellmann, Katja (2011): Emotionale Wirkungen des Erzählens. In: Martinez, Matias (Hg.): Handbuch Erzählliteratur. Theorie, Analyse, Geschichte. Stuttgart, S. 68-74.

Schneider, Irmela (2001): Theorien des Intimen und Privaten. Überlegungen im Anschluss an Richard Sennett und Anthony Giddens. In: Herrmann, Friederike/Lünenborg, Margreth (Hg.): Tabubruch als Programm. Privates und Intimes in den Medien. Opladen, S. 37-48.

7 Die Delegation von Schamgefühlen muss nicht unbedingt bewusste Intention der Autorinnen und Autoren sein. Gut denkbar ist, dass Journalisten eher aus einem Gefühl heraus die Texte in dieser Weise gestalten, weil sie davon ausgehen, dass es bei den Leserinnen und Lesern ankommt. 